Acta Crystallographica Section E

Structure Reports

Online

ISSN 1600-5368

\section{2-Isopropyl-4-methoxy-5-methylphenyl benzoate}

\section{Mohamed Moumou, ${ }^{a}$ Mohamed Akssira, ${ }^{a}$ Ahmed Elhakmaoui, ${ }^{a}$ Lahcen El Ammari, ${ }^{b}$ Ahmed Benharref ${ }^{c}$ and Moha Berraho ${ }^{\mathrm{c}}$ *}

aLaboratoire de Chimie Bioorganique et Analytique, URAC 22, Faculté des Sciences et Techniques, 20800 Mohammedia, Morocco, ${ }^{\mathbf{b}}$ Laboratoire de Chimie du Solide Appliquée, Faculté des Sciences, Avenue Ibn Battouta BP 1014 Rabat, Morocco, and 'Laboratoire de Chimie Biomolécules, Substances Naturelles et Réactivité, URAC 16, Faculté des Sciences Semlalia, BP 2390 Bd My abdellah, 40000 Marrakech, Morocco Correspondence e-mail: mberraho@yahoo.fr

Received 4 March 2010; accepted 11 March 2010

Key indicators: single-crystal X-ray study; $T=298 \mathrm{~K}$; mean $\sigma(\mathrm{C}-\mathrm{C})=0.002 \AA$; $R$ factor $=0.042 ; w R$ factor $=0.119 ;$ data-to-parameter ratio $=15.0$.

The title compound, $\mathrm{C}_{18} \mathrm{H}_{20} \mathrm{O}_{3}$, a hemisynthetic product, was obtained by the reaction of benzoyl chloride and $p$-methoxythymol. The structure comprises two benzene rings bridged by a carboxyl group; the dihedral angle between the rings is $73.54(8)^{\circ}$.

\section{Related literature}

For background to the phytochemical study of Moroccan plants, see: Barrero et al. (2005); Zrira et al. (2005). For background to the medicinal interest in Tetraclinis artculata, from which the title compound was extracted, see: Aitigri et al. (1990).

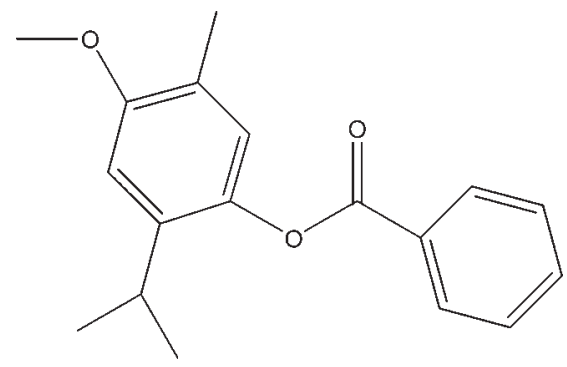

\section{Experimental}

Crystal data

$$
\begin{aligned}
& \mathrm{C}_{18} \mathrm{H}_{20} \mathrm{O}_{3} \\
& M_{r}=284.34 \\
& \text { Monoclinic, } P 2_{b} / n \\
& a=8.4765(4) \AA \\
& b=8.0880(4) \AA \\
& c=23.8119(11) \AA \\
& \beta=98.202(2)^{\circ}
\end{aligned}
$$

$$
\begin{aligned}
& V=1615.80(13) \AA^{3} \\
& Z=4 \\
& \text { Mo } K \alpha \text { radiation } \\
& \mu=0.08 \mathrm{~mm}^{-1} \\
& T=298 \mathrm{~K} \\
& 0.27 \times 0.17 \times 0.12 \mathrm{~mm}
\end{aligned}
$$

\section{Data collection}

Bruker X8 APEX CCD area-

detector diffractometer

14992 measured reflections

2912 independent reflections 2302 reflections with $I>2 \sigma(I)$ $R_{\text {int }}=0.028$

\section{Refinement}

$R\left[F^{2}>2 \sigma\left(F^{2}\right)\right]=0.042$

$w R\left(F^{2}\right)=0.119$

$S=1.02$

2912 reflections

194 parameters

$\mathrm{H}$-atom parameters constrained

$\Delta \rho_{\max }=0.18{\mathrm{e} \AA^{-3}}^{-3}$

$\Delta \rho_{\min }=-0.18{\mathrm{e} \AA^{-3}}^{-3}$

Data collection: APEX2 (Bruker, 2009); cell refinement: SAINTPlus (Bruker, 2009); data reduction: SAINT-Plus; program(s) used to solve structure: SHELXS97 (Sheldrick, 2008); program(s) used to refine structure: SHELXL97 (Sheldrick, 2008); molecular graphics: ORTEP-3 for Windows (Farrugia, 1997); software used to prepare material for publication: Win $G X$ (Farrugia, 1999).

The authors thank the CNRST and RéPAM for financial support and the Unit of Support for Technical and Scientific Research (UATRS, CNRST) for the X-ray diffraction measurements.

Supplementary data and figures for this paper are available from the IUCr electronic archives (Reference: TK2638).

\section{References}

Aitigri, M., Holemen, M., Idriss, M. \& Berrada, M. (1990). Plant. Med. Phytother. 24, 36-43.

Barrero, A. F., Mar Herrador, M., Arteaga, P., Quillez, J., Akssira, M. Mellouki, F. \& Akkad, S. (2005). J. Essent. Oil Res. 17, 166-168.

Bruker (2009). APEX2 and SAINT-Plus. Bruker AXS Inc., Madison, Wisconsin, USA.

Farrugia, L. J. (1997). J. Appl. Cryst. 30, 565.

Farrugia, L. J. (1999). J. Appl. Cryst. 32, 837-838.

Sheldrick, G. M. (2008). Acta Cryst. A64, 112-122.

Zrira, S., Benjilali, B. \& Elamrani, A. (2005). J. Essent. Oil Res. 17, 96-97. 


\section{supporting information}

Acta Cryst. (2010). E66, o850 [doi:10.1107/S160053681000930X]

\section{2-Isopropyl-4-methoxy-5-methylphenyl benzoate}

\section{Mohamed Moumou, Mohamed Akssira, Ahmed Elhakmaoui, Lahcen El Ammari, Ahmed Benharref and Moha Berraho}

\section{S1. Comment}

The title compound, (I), was investigated as a part of our study of essential oils isolated from the sawdust of Tetraclinis articulata originating from the region of Essaouira in Morocco (Aitigri et al., 1990; Barrero et al., 2005; Zrira et al., 2005). In this paper, we present the crystal structure of (I), which was synthesised by the reaction of the benzoyl chloride and $p$-methoxythymol (see Experimental). The molecular structure of (I), Fig. 1, shows the two benzene rings are almost perpendicular with the dihedral angle between them being $73.54(8)^{\circ}$.

\section{S2. Experimental}

The Tetraclinis articulata (Vahl) Masters was collected in the region of Essaouira (Morocco). Wood sawdust was hydrodistilled in a Clevenger-type apparatus for $6 \mathrm{~h}$ to produce essential oils in 3\% yield. The oil was then extracted by diethylether, dried over $\mathrm{Mg}_{2} \mathrm{SO}_{4}$, and the solvent evaporated. The oil was then subjected to silica gel column chromatography by eluting with hexane-ethyl acetate (98:2). The fifth fraction contained $p$-methoxythymol as the major compound. The structure of this product was confirmed by reaction with benzoyl chloride $(0.74 \mathrm{~g}, 5.3 \mathrm{mmol})$ and crude fifth fraction $(0.8$ g) in a solution of $10 \% \mathrm{NaOH}(50 \mathrm{ml})$. The mixture was left under agitation at $298 \mathrm{~K}$ for $1 \mathrm{~h}$. The resulting crystalline precipitate was filtered and recrystallized from methanol. The air-dried crystal $(0.7 \mathrm{~g})$ had a melting point of $259-260 \mathrm{~K}$.

\section{S3. Refinement}

All $\mathrm{H}$ atoms were fixed geometrically and treated as riding with $\mathrm{C}-\mathrm{H}=0.93 \AA$ (aromatic), $0.96 \AA$ (methyl), and $0.98 \AA$ (methine), and with $\mathrm{U}_{\mathrm{iso}}(\mathrm{H})=1.2 \mathrm{U}_{\mathrm{eq}}$ (aromatic, methine) or $\mathrm{U}_{\mathrm{iso}}(\mathrm{H})=1.5 \mathrm{U}_{\mathrm{eq}}$ (methyl). 


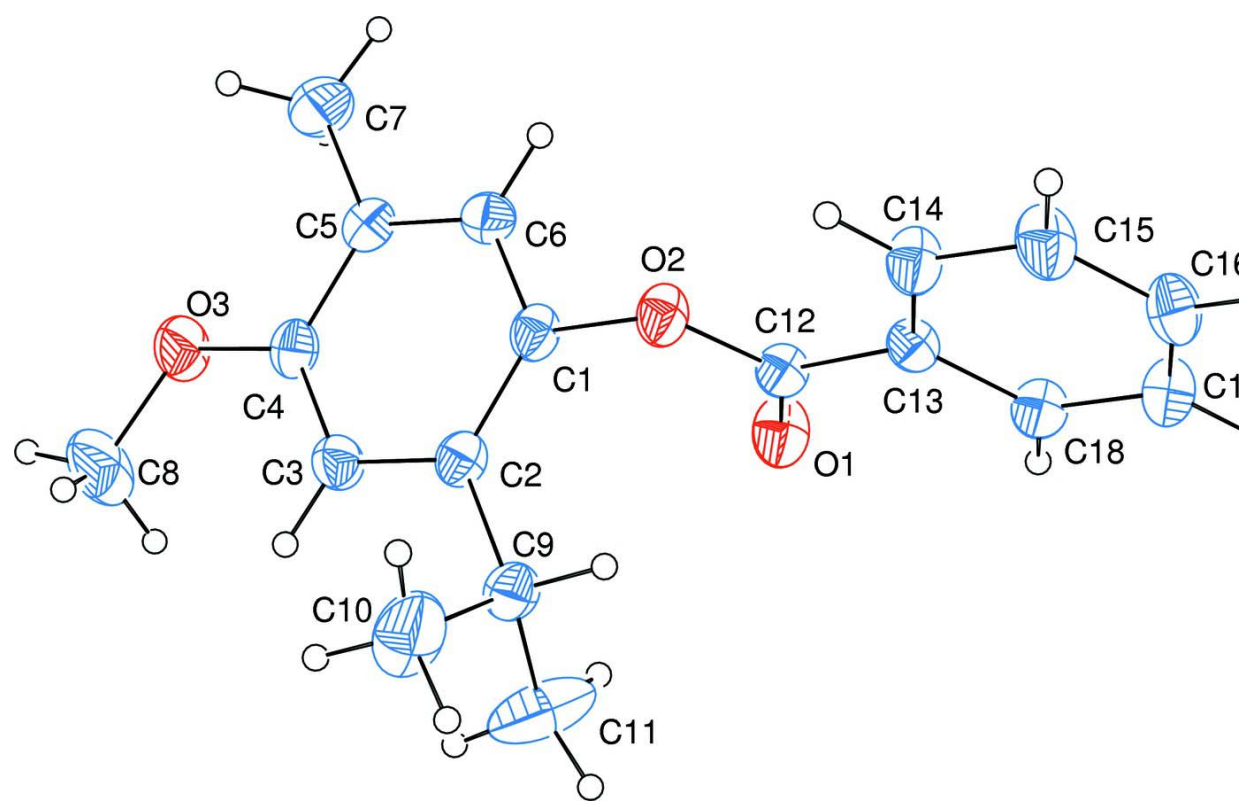

\section{Figure 1}

The molecular structure of (I), showing the atom-labelling scheme. Displacement ellipsoids are drawn at the 30\% probability level. $\mathrm{H}$ atoms are represented as small spheres of arbitrary radii.

\section{2-Isopropyl-4-methoxy-5-methylphenyl benzoate}

Crystal data

$\mathrm{C}_{18} \mathrm{H}_{20} \mathrm{O}_{3}$

$M_{r}=284.34$

Monoclinic, $P 2{ }_{1} / n$

Hall symbol: -P 2yn

$a=8.4765$ (4) $\AA$

$b=8.0880(4) \AA$

$c=23.8119(11) \AA$

$\beta=98.202(2)^{\circ}$

$V=1615.80(13) \AA^{3}$

$Z=4$

Data collection

Bruker X8 APEX CCD area-detector diffractometer

Radiation source: fine-focus sealed tube

Graphite monochromator

$\varphi$ and $\omega$ scans

14992 measured reflections

2912 independent reflections

Refinement

Refinement on $F^{2}$

Least-squares matrix: full

$R\left[F^{2}>2 \sigma\left(F^{2}\right)\right]=0.042$

$w R\left(F^{2}\right)=0.119$

$S=1.02$

2912 reflections
$F(000)=608$

$D_{\mathrm{x}}=1.169 \mathrm{Mg} \mathrm{m}^{-3}$

Mo $K \alpha$ radiation, $\lambda=0.71073 \AA$

Cell parameters from 14992 reflections

$\theta=2.5-25.2^{\circ}$

$\mu=0.08 \mathrm{~mm}^{-1}$

$T=298 \mathrm{~K}$

Prism, colourless

$0.27 \times 0.17 \times 0.12 \mathrm{~mm}$

2302 reflections with $I>2 \sigma(I)$

$R_{\text {int }}=0.028$

$\theta_{\max }=25.2^{\circ}, \theta_{\min }=2.5^{\circ}$

$h=-10 \rightarrow 10$

$k=-9 \rightarrow 9$

$l=-28 \rightarrow 28$

194 parameters

0 restraints

Primary atom site location: structure-invariant direct methods

Secondary atom site location: difference Fourier map 
Hydrogen site location: inferred from neighbouring sites

$\mathrm{H}$-atom parameters constrained

$$
\begin{aligned}
& w=1 /\left[\sigma^{2}\left(F_{\mathrm{o}}{ }^{2}\right)+(0.0574 P)^{2}+0.3991 P\right] \\
& \text { where } P=\left(F_{\mathrm{o}}^{2}+2 F_{\mathrm{c}}{ }^{2}\right) / 3 \\
& (\Delta / \sigma)_{\max }<0.001 \\
& \Delta \rho_{\max }=0.18 \mathrm{e} \AA^{-3} \\
& \Delta \rho_{\min }=-0.18 \mathrm{e} \AA^{-3}
\end{aligned}
$$

\begin{tabular}{|c|c|c|c|c|}
\hline & $x$ & $y$ & $z$ & $U_{\text {iso }} * / U_{\text {eq }}$ \\
\hline $\mathrm{C} 1$ & $0.77989(18)$ & $0.64673(18)$ & $0.08713(6)$ & $0.0432(4)$ \\
\hline $\mathrm{C} 2$ & $0.69644(17)$ & $0.56847(18)$ & $0.12520(6)$ & $0.0430(4)$ \\
\hline $\mathrm{C} 3$ & $0.62554(18)$ & 0.41749 (19) & $0.10781(6)$ & $0.0474(4)$ \\
\hline H3 & 0.5675 & 0.3612 & 0.1321 & $0.057^{*}$ \\
\hline $\mathrm{C} 4$ & $0.63938(18)$ & 0.34959 (19) & $0.05553(7)$ & $0.0467(4)$ \\
\hline $\mathrm{C} 5$ & $0.72412(18)$ & $0.4313(2)$ & $0.01763(6)$ & $0.0471(4)$ \\
\hline C6 & $0.79271(19)$ & $0.5809(2)$ & $0.03449(6)$ & $0.0483(4)$ \\
\hline H6 & 0.8487 & 0.6387 & 0.0099 & $0.058 *$ \\
\hline $\mathrm{C} 7$ & $0.7392(3)$ & $0.3562(3)$ & $-0.03917(7)$ & $0.0707(5)$ \\
\hline H7A & 0.7937 & 0.4320 & -0.0608 & $0.106^{*}$ \\
\hline H7B & 0.6349 & 0.3338 & -0.0592 & $0.106^{*}$ \\
\hline $\mathrm{H} 7 \mathrm{C}$ & 0.7985 & 0.2550 & -0.0338 & $0.106^{*}$ \\
\hline $\mathrm{C} 8$ & $0.4850(3)$ & $0.1124(3)$ & $0.07224(11)$ & $0.0960(8)$ \\
\hline H8A & 0.5503 & 0.0867 & 0.1075 & $0.144 *$ \\
\hline H8B & 0.4464 & 0.0117 & 0.0538 & $0.144^{*}$ \\
\hline $\mathrm{H} 8 \mathrm{C}$ & 0.3963 & 0.1789 & 0.0795 & $0.144^{*}$ \\
\hline $\mathrm{C} 9$ & $0.6813(2)$ & $0.6391(2)$ & $0.18320(6)$ & $0.0508(4)$ \\
\hline H9 & 0.7425 & 0.7423 & 0.1867 & $0.061^{*}$ \\
\hline $\mathrm{C} 10$ & $0.5123(3)$ & $0.6843(4)$ & $0.18816(10)$ & $0.0985(9)$ \\
\hline $\mathrm{H} 10 \mathrm{~A}$ & 0.4737 & 0.7620 & 0.1589 & $0.148 *$ \\
\hline H10B & 0.5078 & 0.7332 & 0.2246 & $0.148^{*}$ \\
\hline $\mathrm{H} 10 \mathrm{C}$ & 0.4472 & 0.5868 & 0.1841 & $0.148 *$ \\
\hline C11 & $0.7565(4)$ & 0.5276 & $0.22999(9)$ & $0.1153(10)$ \\
\hline H11A & 0.6928 & 0.4298 & 0.2311 & $0.173 *$ \\
\hline H11B & 0.7631 & 0.5845 & 0.2656 & $0.173^{*}$ \\
\hline $\mathrm{H} 11 \mathrm{C}$ & 0.8616 & 0.4973 & 0.2232 & $0.173^{*}$ \\
\hline $\mathrm{C} 12$ & 0.99177 (17) & $0.80615(17)$ & $0.13445(6)$ & $0.0416(3)$ \\
\hline C13 & $1.05364(17)$ & $0.97651(17)$ & $0.14447(6)$ & $0.0402(3)$ \\
\hline C14 & $0.9796(2)$ & $1.11338(19)$ & $0.11761(7)$ & $0.0535(4)$ \\
\hline H14 & 0.8884 & 1.1002 & 0.0912 & $0.064 *$ \\
\hline $\mathrm{C} 15$ & $1.0407(2)$ & $1.2690(2)$ & $0.12988(8)$ & $0.0605(5)$ \\
\hline $\mathrm{H} 15$ & 0.9906 & 1.3607 & 0.1118 & $0.073^{*}$ \\
\hline C16 & $1.1752(2)$ & $1.2895(2)$ & $0.16867(8)$ & $0.0561(4)$ \\
\hline H16 & 1.2156 & 1.3950 & 0.1769 & $0.067 *$ \\
\hline $\mathrm{C} 17$ & $1.2503(2)$ & $1.1547(2)$ & $0.19528(8)$ & $0.0561(4)$ \\
\hline H17 & 1.3419 & 1.1688 & 0.2214 & $0.067 *$ \\
\hline C18 & $1.19010(18)$ & 0.99813 (19) & $0.18335(7)$ & $0.0478(4)$ \\
\hline H18 & 1.2412 & 0.9069 & 0.2014 & $0.057^{*}$ \\
\hline $\mathrm{O} 1$ & $1.05685(14)$ & $0.68399(13)$ & $0.15440(5)$ & 0.0593 (3) \\
\hline
\end{tabular}

Fractional atomic coordinates and isotropic or equivalent isotropic displacement parameters $\left(\AA^{2}\right)$ 
supporting information

\begin{tabular}{lllll}
$\mathrm{O} 2$ & $0.85023(13)$ & $0.80333(12)$ & $0.10003(5)$ & $0.0495(3)$ \\
$\mathrm{O} 3$ & $0.57625(15)$ & $0.20040(15)$ & $0.03683(5)$ & $0.0644(4)$ \\
\hline
\end{tabular}

Atomic displacement parameters $\left(\AA^{2}\right)$

\begin{tabular}{lllllll}
\hline & $U^{11}$ & $U^{22}$ & $U^{33}$ & $U^{12}$ & $U^{13}$ & $U^{23}$ \\
\hline C1 & $0.0463(8)$ & $0.0325(8)$ & $0.0480(8)$ & $-0.0015(6)$ & $-0.0032(7)$ & $0.0000(6)$ \\
C2 & $0.0447(8)$ & $0.0382(8)$ & $0.0449(8)$ & $0.0017(6)$ & $0.0021(6)$ & $-0.0034(6)$ \\
C3 & $0.0504(9)$ & $0.0436(9)$ & $0.0490(9)$ & $-0.0072(7)$ & $0.0095(7)$ & $-0.0038(7)$ \\
C4 & $0.0465(8)$ & $0.0400(8)$ & $0.0523(9)$ & $-0.0046(7)$ & $0.0025(7)$ & $-0.0093(7)$ \\
C5 & $0.0486(9)$ & $0.0496(9)$ & $0.0416(8)$ & $-0.0003(7)$ & $0.0011(7)$ & $-0.0046(7)$ \\
C6 & $0.0525(9)$ & $0.0475(9)$ & $0.0440(8)$ & $-0.0030(7)$ & $0.0035(7)$ & $0.0057(7)$ \\
C7 & $0.0856(14)$ & $0.0759(13)$ & $0.0517(10)$ & $-0.0091(11)$ & $0.0138(9)$ & $-0.0162(9)$ \\
C8 & $0.123(2)$ & $0.0693(14)$ & $0.1051(17)$ & $-0.0538(14)$ & $0.0470(15)$ & $-0.0320(13)$ \\
C9 & $0.0592(10)$ & $0.0455(9)$ & $0.0476(9)$ & $-0.0051(7)$ & $0.0069(7)$ & $-0.0086(7)$ \\
C10 & $0.0700(13)$ & $0.142(2)$ & $0.0866(15)$ & $-0.0023(14)$ & $0.0221(12)$ & $-0.0558(16)$ \\
C11 & $0.199(3)$ & $0.0939(18)$ & $0.0466(11)$ & $0.0372(19)$ & $-0.0033(15)$ & $0.0001(11)$ \\
C12 & $0.0446(8)$ & $0.0349(8)$ & $0.0449(8)$ & $0.0028(6)$ & $0.0046(6)$ & $0.0006(6)$ \\
C13 & $0.0428(8)$ & $0.0335(7)$ & $0.0449(8)$ & $0.0013(6)$ & $0.0087(6)$ & $0.0001(6)$ \\
C14 & $0.0505(9)$ & $0.0384(9)$ & $0.0673(10)$ & $-0.0001(7)$ & $-0.0062(8)$ & $0.0053(8)$ \\
C15 & $0.0617(11)$ & $0.0332(8)$ & $0.0836(13)$ & $-0.0006(7)$ & $-0.0003(9)$ & $0.0082(8)$ \\
C16 & $0.0568(10)$ & $0.0375(9)$ & $0.0741(11)$ & $-0.0090(7)$ & $0.0100(9)$ & $-0.0060(8)$ \\
C17 & $0.0490(9)$ & $0.0526(10)$ & $0.0641(10)$ & $-0.0059(8)$ & $-0.0008(8)$ & $-0.0078(8)$ \\
C18 & $0.0485(9)$ & $0.0405(9)$ & $0.0531(9)$ & $0.0044(7)$ & $0.0030(7)$ & $0.0015(7)$ \\
O1 & $0.0596(7)$ & $0.0331(6)$ & $0.0803(8)$ & $0.0040(5)$ & $-0.0070(6)$ & $0.0040(5)$ \\
O2 & $0.0540(6)$ & $0.0319(5)$ & $0.0583(7)$ & $-0.0037(5)$ & $-0.0064(5)$ & $0.0031(5)$ \\
O3 & $0.0756(8)$ & $0.0526(7)$ & $0.0670(8)$ & $-0.0224(6)$ & $0.0165(6)$ & $-0.0217(6)$ \\
& & & & & & \\
\hline
\end{tabular}

Geometric parameters $\left(\AA,^{\circ}\right)$

\begin{tabular}{llll}
\hline $\mathrm{C} 1-\mathrm{C} 2$ & $1.380(2)$ & $\mathrm{C} 9-\mathrm{H} 9$ & 0.9800 \\
$\mathrm{C} 1-\mathrm{C} 6$ & $1.380(2)$ & $\mathrm{C} 10-\mathrm{H} 10 \mathrm{~A}$ & 0.9600 \\
$\mathrm{C} 1-\mathrm{O} 2$ & $1.4149(17)$ & $\mathrm{C} 10-\mathrm{H} 10 \mathrm{~B}$ & 0.9600 \\
$\mathrm{C} 2-\mathrm{C} 3$ & $1.397(2)$ & $\mathrm{C} 10-\mathrm{H} 10 \mathrm{C}$ & 0.9600 \\
$\mathrm{C} 2-\mathrm{C} 9$ & $1.517(2)$ & $\mathrm{C} 11-\mathrm{H} 11 \mathrm{~A}$ & 0.9600 \\
$\mathrm{C} 3-\mathrm{C} 4$ & $1.381(2)$ & $\mathrm{C} 11-\mathrm{H} 11 \mathrm{~B}$ & 0.9600 \\
$\mathrm{C} 3-\mathrm{H} 3$ & 0.9300 & $\mathrm{C} 11-\mathrm{H} 11 \mathrm{C}$ & 0.9600 \\
$\mathrm{C} 4-\mathrm{O} 3$ & $1.3685(18)$ & $\mathrm{C} 12-\mathrm{O} 1$ & $1.1964(17)$ \\
$\mathrm{C} 4-\mathrm{C} 5$ & $1.397(2)$ & $\mathrm{C} 12-\mathrm{O} 2$ & $1.3531(18)$ \\
$\mathrm{C} 5-\mathrm{C} 6$ & $1.378(2)$ & $\mathrm{C} 12-\mathrm{C} 13$ & $1.481(2)$ \\
$\mathrm{C} 5-\mathrm{C} 7$ & $1.504(2)$ & $\mathrm{C} 13-\mathrm{C} 14$ & $1.384(2)$ \\
$\mathrm{C} 6-\mathrm{H} 6$ & 0.9300 & $\mathrm{C} 13-\mathrm{C} 18$ & $1.386(2)$ \\
$\mathrm{C} 7-\mathrm{H} 7 \mathrm{~A}$ & 0.9600 & $\mathrm{C} 14-\mathrm{C} 15$ & $1.377(2)$ \\
$\mathrm{C} 7-\mathrm{H} 7 \mathrm{~B}$ & 0.9600 & $\mathrm{C} 14-\mathrm{H} 14$ & 0.9300 \\
$\mathrm{C} 7-\mathrm{H} 7 \mathrm{C}$ & 0.9600 & $\mathrm{C} 15-\mathrm{C} 16$ & $1.371(2)$ \\
$\mathrm{C} 8-\mathrm{O} 3$ & $1.415(2)$ & $\mathrm{C} 15-\mathrm{H} 15$ & 0.9300 \\
$\mathrm{C} 8-\mathrm{H} 8 \mathrm{~A}$ & 0.9600 & $\mathrm{C} 16-\mathrm{C} 17$ & $1.371(2)$ \\
$\mathrm{C} 8-\mathrm{H} 8 \mathrm{~B}$ & 0.9600 & $\mathrm{C} 16-\mathrm{H} 16$ & 0.9300
\end{tabular}




\begin{tabular}{|c|c|c|c|}
\hline $\mathrm{C} 8-\mathrm{H} 8 \mathrm{C}$ & 0.9600 & $\mathrm{C} 17-\mathrm{C} 18$ & $1.379(2)$ \\
\hline $\mathrm{C} 9-\mathrm{C} 10$ & $1.500(3)$ & $\mathrm{C} 17-\mathrm{H} 17$ & 0.9300 \\
\hline $\mathrm{C} 9-\mathrm{C} 11$ & $1.503(3)$ & $\mathrm{C} 18-\mathrm{H} 18$ & 0.9300 \\
\hline $\mathrm{C} 2-\mathrm{C} 1-\mathrm{C} 6$ & $122.25(14)$ & $\mathrm{C} 9-\mathrm{C} 10-\mathrm{H} 10 \mathrm{~A}$ & 109.5 \\
\hline $\mathrm{C} 2-\mathrm{C} 1-\mathrm{O} 2$ & $120.49(13)$ & $\mathrm{C} 9-\mathrm{C} 10-\mathrm{H} 10 \mathrm{~B}$ & 109.5 \\
\hline $\mathrm{C} 6-\mathrm{C} 1-\mathrm{O} 2$ & $117.19(13)$ & $\mathrm{H} 10 \mathrm{~A}-\mathrm{C} 10-\mathrm{H} 10 \mathrm{~B}$ & 109.5 \\
\hline $\mathrm{C} 1-\mathrm{C} 2-\mathrm{C} 3$ & $116.42(14)$ & $\mathrm{C} 9-\mathrm{C} 10-\mathrm{H} 10 \mathrm{C}$ & 109.5 \\
\hline $\mathrm{C} 1-\mathrm{C} 2-\mathrm{C} 9$ & $122.90(14)$ & $\mathrm{H} 10 \mathrm{~A}-\mathrm{C} 10-\mathrm{H} 10 \mathrm{C}$ & 109.5 \\
\hline $\mathrm{C} 3-\mathrm{C} 2-\mathrm{C} 9$ & $120.68(14)$ & $\mathrm{H} 10 \mathrm{~B}-\mathrm{C} 10-\mathrm{H} 10 \mathrm{C}$ & 109.5 \\
\hline $\mathrm{C} 4-\mathrm{C} 3-\mathrm{C} 2$ & $121.77(15)$ & $\mathrm{C} 9-\mathrm{C} 11-\mathrm{H} 11 \mathrm{~A}$ & 109.5 \\
\hline $\mathrm{C} 4-\mathrm{C} 3-\mathrm{H} 3$ & 119.1 & $\mathrm{C} 9-\mathrm{C} 11-\mathrm{H} 11 \mathrm{~B}$ & 109.5 \\
\hline $\mathrm{C} 2-\mathrm{C} 3-\mathrm{H} 3$ & 119.1 & $\mathrm{H} 11 \mathrm{~A}-\mathrm{C} 11-\mathrm{H} 11 \mathrm{~B}$ & 109.5 \\
\hline $\mathrm{O} 3-\mathrm{C} 4-\mathrm{C} 3$ & $124.36(14)$ & $\mathrm{C} 9-\mathrm{C} 11-\mathrm{H} 11 \mathrm{C}$ & 109.5 \\
\hline $\mathrm{O} 3-\mathrm{C} 4-\mathrm{C} 5$ & $114.83(13)$ & $\mathrm{H} 11 \mathrm{~A}-\mathrm{C} 11-\mathrm{H} 11 \mathrm{C}$ & 109.5 \\
\hline $\mathrm{C} 3-\mathrm{C} 4-\mathrm{C} 5$ & $120.80(14)$ & $\mathrm{H} 11 \mathrm{~B}-\mathrm{C} 11-\mathrm{H} 11 \mathrm{C}$ & 109.5 \\
\hline $\mathrm{C} 6-\mathrm{C} 5-\mathrm{C} 4$ & $117.50(14)$ & $\mathrm{O} 1-\mathrm{C} 12-\mathrm{O} 2$ & $123.04(13)$ \\
\hline $\mathrm{C} 6-\mathrm{C} 5-\mathrm{C} 7$ & $122.01(15)$ & $\mathrm{O} 1-\mathrm{C} 12-\mathrm{C} 13$ & $124.86(14)$ \\
\hline $\mathrm{C} 4-\mathrm{C} 5-\mathrm{C} 7$ & $120.49(15)$ & $\mathrm{O} 2-\mathrm{C} 12-\mathrm{C} 13$ & $112.10(12)$ \\
\hline $\mathrm{C} 5-\mathrm{C} 6-\mathrm{C} 1$ & $121.25(14)$ & $\mathrm{C} 14-\mathrm{C} 13-\mathrm{C} 18$ & $119.23(14)$ \\
\hline $\mathrm{C} 5-\mathrm{C} 6-\mathrm{H} 6$ & 119.4 & $\mathrm{C} 14-\mathrm{C} 13-\mathrm{C} 12$ & $122.88(13)$ \\
\hline $\mathrm{C} 1-\mathrm{C} 6-\mathrm{H} 6$ & 119.4 & $\mathrm{C} 18-\mathrm{C} 13-\mathrm{C} 12$ & $117.88(13)$ \\
\hline $\mathrm{C} 5-\mathrm{C} 7-\mathrm{H} 7 \mathrm{~A}$ & 109.5 & $\mathrm{C} 15-\mathrm{C} 14-\mathrm{C} 13$ & $120.07(15)$ \\
\hline $\mathrm{C} 5-\mathrm{C} 7-\mathrm{H} 7 \mathrm{~B}$ & 109.5 & $\mathrm{C} 15-\mathrm{C} 14-\mathrm{H} 14$ & 120.0 \\
\hline $\mathrm{H} 7 \mathrm{~A}-\mathrm{C} 7-\mathrm{H} 7 \mathrm{~B}$ & 109.5 & $\mathrm{C} 13-\mathrm{C} 14-\mathrm{H} 14$ & 120.0 \\
\hline $\mathrm{C} 5-\mathrm{C} 7-\mathrm{H} 7 \mathrm{C}$ & 109.5 & $\mathrm{C} 16-\mathrm{C} 15-\mathrm{C} 14$ & $120.35(16)$ \\
\hline $\mathrm{H} 7 \mathrm{~A}-\mathrm{C} 7-\mathrm{H} 7 \mathrm{C}$ & 109.5 & $\mathrm{C} 16-\mathrm{C} 15-\mathrm{H} 15$ & 119.8 \\
\hline $\mathrm{H} 7 \mathrm{~B}-\mathrm{C} 7-\mathrm{H} 7 \mathrm{C}$ & 109.5 & $\mathrm{C} 14-\mathrm{C} 15-\mathrm{H} 15$ & 119.8 \\
\hline $\mathrm{O} 3-\mathrm{C} 8-\mathrm{H} 8 \mathrm{~A}$ & 109.5 & $\mathrm{C} 15-\mathrm{C} 16-\mathrm{C} 17$ & $120.11(15)$ \\
\hline $\mathrm{O} 3-\mathrm{C} 8-\mathrm{H} 8 \mathrm{~B}$ & 109.5 & $\mathrm{C} 15-\mathrm{C} 16-\mathrm{H} 16$ & 119.9 \\
\hline $\mathrm{H} 8 \mathrm{~A}-\mathrm{C} 8-\mathrm{H} 8 \mathrm{~B}$ & 109.5 & $\mathrm{C} 17-\mathrm{C} 16-\mathrm{H} 16$ & 119.9 \\
\hline $\mathrm{O} 3-\mathrm{C} 8-\mathrm{H} 8 \mathrm{C}$ & 109.5 & $\mathrm{C} 16-\mathrm{C} 17-\mathrm{C} 18$ & $120.07(15)$ \\
\hline $\mathrm{H} 8 \mathrm{~A}-\mathrm{C} 8-\mathrm{H} 8 \mathrm{C}$ & 109.5 & $\mathrm{C} 16-\mathrm{C} 17-\mathrm{H} 17$ & 120.0 \\
\hline $\mathrm{H} 8 \mathrm{~B}-\mathrm{C} 8-\mathrm{H} 8 \mathrm{C}$ & 109.5 & $\mathrm{C} 18-\mathrm{C} 17-\mathrm{H} 17$ & 120.0 \\
\hline $\mathrm{C} 10-\mathrm{C} 9-\mathrm{C} 11$ & $113.4(2)$ & $\mathrm{C} 17-\mathrm{C} 18-\mathrm{C} 13$ & $120.16(14)$ \\
\hline $\mathrm{C} 10-\mathrm{C} 9-\mathrm{C} 2$ & $111.69(14)$ & $\mathrm{C} 17-\mathrm{C} 18-\mathrm{H} 18$ & 119.9 \\
\hline $\mathrm{C} 11-\mathrm{C} 9-\mathrm{C} 2$ & $111.55(15)$ & $\mathrm{C} 13-\mathrm{C} 18-\mathrm{H} 18$ & 119.9 \\
\hline $\mathrm{C} 10-\mathrm{C} 9-\mathrm{H} 9$ & 106.6 & $\mathrm{C} 12-\mathrm{O} 2-\mathrm{C} 1$ & $117.16(11)$ \\
\hline $\mathrm{C} 11-\mathrm{C} 9-\mathrm{H} 9$ & 106.6 & $\mathrm{C} 4-\mathrm{O} 3-\mathrm{C} 8$ & $118.13(13)$ \\
\hline $\mathrm{C} 2-\mathrm{C} 9-\mathrm{H} 9$ & 106.6 & & \\
\hline
\end{tabular}

